\title{
Simultaneous Binding of Two Protein Kinases to a Calcium- Dependent Potassium Channel
}

\author{
Jing Wang, Yi Zhou, Hua Wen, and Irwin B. Levitan \\ Department of Biochemistry and Volen Center for Complex Systems, Brandeis University, \\ Waltham, Massachusetts 02454
}

Large-conductance calcium-dependent potassium channels are subject to modulation by protein kinases, phosphatases, and other signaling proteins, and it has been inferred from electrophysiological experiments that signaling proteins sometimes can be intimately associated with these channels in a regulatory complex. We show here that endogenous protein kinase activity coimmunoprecipitates with both native and recombinant Drosophila Slowpoke (dSlo) calcium-dependent potassium channels. Coimmunoprecipitation experiments using antibodies against several protein kinases demonstrate that dSlo can bind simultaneously to the Src tyrosine kinase and to the catalytic subunit of the cAMP-dependent protein kinase (PKAc). Both kinases can phosphorylate the channel in Dro- sophila heads and in heterologous host cells. The PKAc binds directly to a 172-amino acid region in the C-terminal domain of dSlo, without the intervention of regulatory subunits or anchoring proteins, and channel phosphorylation by PKAc is not required for this binding interaction. In contrast, several phosphorylatable tyrosine residues in dSlo are important for Src binding. The results are consistent with the idea that an ion channel can act as a scaffold for its own specific set of modulatory enzymes.

Key words: potassium channel; modulation; protein kinase; channel phosphorylation; channel-binding protein, Slowpoke; Drosophila
Large-conductance calcium-dependent potassium channels are ubiquitous in nerve, muscle, and other cell types (Latorre et al., 1989). Modulation of these channels, most notably by protein phosphorylation (Levitan, 1994), can influence such cellular functions as neurotransmitter release, hormone secretion, and muscle contraction. Calcium-dependent potassium channels play a particularly important role in neuronal signaling, because they respond to both the intracellular calcium concentration and the membrane potential. Hence these channels provide a critical link between intracellular biochemical messenger systems and the electrical properties of the plasma membrane.

Previous functional studies of calcium-dependent potassium channels, from rat brain and other tissues, suggested that protein kinase and phosphatase activities might be closely associated with these channels (Chung et al., 1991; White et al., 1993; Bielefeldt and Jackson, 1994; Lee et al., 1995; Reinhart and Levitan, 1995; Tian et al., 1998). This conclusion was inferred from experiments in which channels in isolated membrane patches or in artificial lipid bilayers could be modulated via phosphorylation and dephosphorylation, without the addition of an exogenous protein kinase or phosphatase. More recent biochemical experiments have demonstrated more directly the binding of protein tyrosine kinases to several other types of ion channels (Swope and Huganir, 1994; Fuhrer and Hall, 1996; Holmes et al., 1996; Yu et al.,

\footnotetext{
Received Oct. 6, 1998; revised Feb. 9, 1999; accepted Feb 17, 1999.

This work was supported by a grant from the National Institutes of Health (to I.B.L.). We thank Jack Riordan and Rick Huganir for PKAc and Src constructs and Leslie Griffith, Todd Holmes, Chris Miller, and Deborah Sodickson for critical comments on this manuscript.

Correspondence should be addressed to Irwin B. Levitan, Volen Center for Complex Systems, Brandeis University, 415 South Street, Waltham, MA 02454.

Dr. Wang's present address: DNAX Research Institute, 901 California Avenue, Palo Alto, CA 94304.

Copyright (C) 1999 Society for Neuroscience $\quad 0270-6474 / 99 / 190001-\bullet \$ 05.00 / 0$
}

1997), in several cases via specific and well characterized proteinprotein interaction domains (Swope and Huganir, 1994; Holmes et al., 1996).

We show here using a coimmunoprecipitation-Western blot strategy that the Drosophila Slowpoke calcium-dependent potassium channel dSlo (Atkinson et al., 1991; Adelman et al., 1992) can bind simultaneously to two different protein kinases, one a serine/threonine kinase and the other a tyrosine kinase. Both of the bound kinases can phosphorylate the channel. We also demonstrate that one of these kinases, the cAMP-dependent protein kinase catalytic subunit (PKAc), can interact with a portion of the channel protein directly rather than via regulatory subunits or anchoring proteins. These results suggest that the dSlo channel itself can act as a scaffold for its own specific set of modulatory enzymes. The demonstration that an ion channel can participate in a regulatory complex with multiple signaling proteins has fundamental implications for neuronal function.

\section{MATERIALS AND METHODS}

Antibody generation. The anti-dSlo antibody was generated by immunizing rabbits with a Gene 9 fusion protein (Park et al., 1991) containing the

This article is published in The Journal of Neuroscience, Rapid Communications Section, which publishes brief, peerreviewed papers online, not in print. Rapid Communications are posted online approximately one month earlier than they would appear if printed. They are listed in the Table of Contents of the next open issue of JNeurosci. Cite this article as: JNeurosci, 1999, 19:RC4 (1-7). The publication date is the date of posting online at www.jneurosci.org.

http://www.jneurosci.org/cgi/content/full/2982 
C-terminal 58 amino acids of dSlo (splice variant, A1C2E1G3I0; for numbering, see Adelman et al., 1992). Specific anti-dSlo antibody was purified (Harlow and Lane, 1988) using an affinity column containing a maltose-binding protein (New England Biolabs, Beverly, MA) fused with the same dSlo C-terminal sequence. The method used to generate the sequence- and phosphorylation-specific antibody anti-pS942 was similar to that of Patton et al. (1991). Details of the preparation, purification, and characterization of both antibodies will be described elsewhere (J. Wang, Y. Zhou, H. Wen, and I. B. Levitan, manuscript in preparation).

Transfection and immunoprecipitation. ts A201 cells were maintained in DMEM supplemented with 10\% fetal bovine serum (Life Technologies, Gaithersburg, MD). A calcium phosphate transfection protocol was used as described (Sambrook et al., 1989), with cDNAs subcloned into pcDNA3 (Invitrogen, San Diego, CA). For immunoprecipitation, transfected cells were lysed or fly heads were homogenized in lysis buffer containing $1 \%$ 3-[(3-cholamidopropyl)dimethylammonio]-1propanesulfonic acid, $20 \mathrm{~mm}$ Tris- $\mathrm{Cl}, \mathrm{pH} 7.5,10 \mathrm{~mm}$ EDTA, $120 \mathrm{~mm}$ $\mathrm{NaCl}, 50 \mathrm{~mm} \mathrm{KCl}, 2 \mathrm{~mm}$ DTT, and protease inhibitors (1 mM PMSF and $1 \mu \mathrm{g} / \mathrm{ml}$ aprotonin, leupeptin, and pepstatin A). The lysates, with $\sim 1$ $\mathrm{mg} / \mathrm{ml}$ solublized protein, were precleared with $20 \mu \mathrm{l} / \mathrm{ml}$ protein A-agarose (Santa Cruz Biotechnology, Santa Cruz, CA) and with preimmune rabbit serum for $1 \mathrm{hr}$. dSlo, PKAc, or Src was immunoprecipitated for $2 \mathrm{hr}$ at $4^{\circ} \mathrm{C}$ with $2 \mu \mathrm{g} / \mathrm{ml}$ anti-dSlo, $5 \mu \mathrm{g} / \mathrm{ml}$ anti-PKAc (Santa Cruz), or $5 \mu \mathrm{g} / \mathrm{ml}$ anti-Src (Oncogene Science, Uniondale, NY), respectively, together with $20 \mu \mathrm{l} / \mathrm{ml}$ protein A-agarose. The immunoprecipitates were then washed with lysis buffer five times.

Western blot. Proteins in the cell lysates or immunoprecipitates were separated on a $7.5 \%$ polyacrylamide gel and transferred to a nitrocellulose membrane that was blocked with $5 \%$ nonfat milk in $10 \mathrm{~mm}$ Tris-Cl, pH 7.5, $150 \mathrm{~mm} \mathrm{NaCl}$, and $0.1 \%$ Tween 20 (TBST). The membrane was then incubated with appropriate primary antibody $(0.5 \mu \mathrm{g} / \mathrm{ml}$ anti-dSlo or $1 \mu \mathrm{g} / \mathrm{ml}$ anti-Src, anti-phosphotyrosine, or anti-PKAc) in blocking buffer at $4^{\circ} \mathrm{C}$ overnight. After three washes with TBST, the membrane was incubated with a 1:3000 dilution of horseradish peroxidaseconjugated donkey anti-rabbit IgG (Amersham, Arlington Heights, IL) for $1 \mathrm{hr}$ at room temperature. Protein complexes were visualized with ECL (Amersham) after three washes of the membrane with TBST and one wash with TBS.

In vitro kinase assay. dSlo immunoprecipitates were washed three times with kinase assay buffer (50 mm Tris-Cl, $\mathrm{pH}$ 7.4, $0.1 \mathrm{~mm}$ EDTA, 10 $\mathrm{mM} \mathrm{MgCl} l_{2}, 10 \mathrm{mM} \mathrm{MnCl}_{2}$, and $2 \mathrm{~mm} \mathrm{DTT}$ ) and then resuspended in this buffer. Kinase activity in the immunoprecipitate, which can phosphorylate the immunoprecipitated dSlo, was assayed in vitro. The kinase reactions were initiated with $1 \mu \mathrm{M}$ ATP $\left(5 \mu \mathrm{Ci}\right.$ of $\left.\left[\gamma^{-}{ }^{32} \mathrm{P}\right] \mathrm{ATP}\right)$ and quenched with $2 \times$ SDS loading buffer (Sambrook et al., 1989) at the end of a 30 min incubation. Proteins were separated on 7.5\% SDS gels, and ${ }^{32} \mathrm{P}$-labeled phosphoproteins were visualized on autoradiographs.

In-gel kinase assay. To further characterize the protein kinase activity in immunoprecipitates, an in-gel kinase assay (Chen and Pan, 1994) was used. The in-gel substrate was a glutathione $S$-transferase (GST) fusion protein containing the dSlo amino acid sequence from residues 821 to 993 (GST-A). A 7.5\% SDS polyacrylamide gel was prepared with 0.2 $\mathrm{mg} / \mathrm{ml}$ GST-A copolymerized in the gel. After the electrophoresis of anti-dSlo immunoprecipitates prepared from vector- or dSlo-transfected cells, the gel was processed as described (Chen and Pan, 1994), except that the modified kinase assay buffer was used (see above).

Protein overlay assay. PKAc was biotinylated with a protein biotinylation kit from Boehringer Mannheim (Indianapolis, IN). GST fusion proteins (see Results) were separated on a $10 \%$ SDS polyacrylamide gel and transferred to a nitrocellulose membrane. After blocking with 5\% nonfat milk in TBST, the membrane was incubated with biotinylated PKAc $(\sim 2 \mu \mathrm{g} / \mathrm{ml})$ in blocking buffer for $1 \mathrm{hr}$ at room temperature. After three washes with TBST, the membrane was incubated with HRPconjugated streptavidin (Amersham) at a 1:1500 dilution in blocking buffer for $1 \mathrm{hr}$. The bound protein was visualized with ECL after three washes with TBST and one wash with TBS.

\section{RESULTS}

\section{Endogenous protein kinase activity coimmunoprecipitates with native and recombinant dSlo}

The dSlo calcium-dependent potassium channel can be phosphorylated in vitro by several different exogenous protein kinases, including PKA, protein kinase $\mathrm{C}$, calcium/calmodulin-dependent
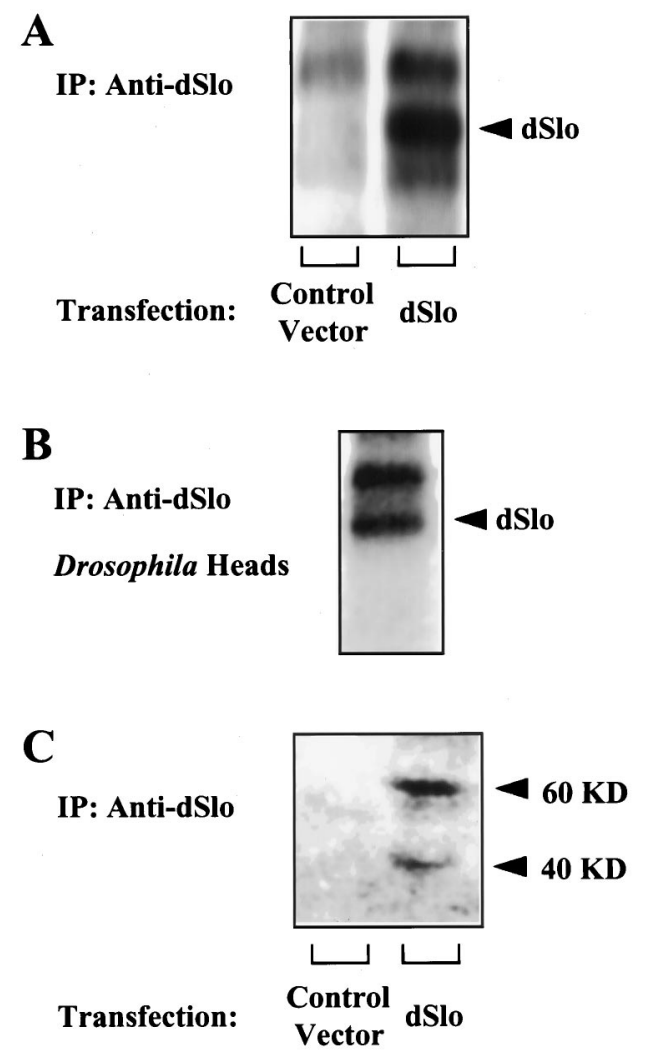

Figure 1. Protein kinase activity that coimmunoprecipitates with dSlo. $(A)$ Phosphorylation of dSlo in vitro by kinase activity in dSlo immunoprecipitates from tsA201 cells (Margolskee et al., 1993) that had been transfected with control vector or dSlo. (B) Phosphorylation of a $130 \mathrm{kDa}$ band in vitro by kinase activity in dSlo immunoprecipitates from Drosophila heads. In addition to the $130 \mathrm{kDa}$ band that corresponds in molecular weight to dSlo (arrows), a higher molecular weight band from both tsA201 cells and Drosophila heads is recognized and immunoprecipitated by anti-dSlo and is a substrate in vitro for the co-immunoprecipitated kinase activity. $(C)$ In-gel kinase assay of dSlo immunoprecipitates prepared from control vector- or dSlo-transfected cells.

protein kinase II (CaMKII), and Src tyrosine kinase (Wang, Zhou, Wen, and Levitan, manuscript in preparation). To test whether any endogenous kinase is bound physically to the channel in vivo, we expressed dSlo cDNA in tsA201 cells (Margolskee et al., 1993) and measured protein kinase activity in a dSlo immunoprecipitate. Endogenous protein kinase activity from the tsA201 cells coimmunoprecipitates with dSlo and can phosphorylate the channel (Fig. $1 A$ ). Similarly, native kinase activity from Drosophila heads coimmunoprecipitates with native dSlo and can phosphorylate several proteins in the immunoprecipitate, including one of molecular weight corresponding to that of dSlo (Fig. $1 B)$. To characterize further the endogenous kinase(s) associated with the channel, we performed an in-gel kinase assay (Chen and Pan, 1994), using as the substrate a GST fusion protein containing the dSlo amino acid sequence from residues 821 to 993 (GST-A). When a dSlo immunoprecipitate from dSlo-transfected tsA201 cells is separated on an SDS gel polymerized with GST-A, and the gel is incubated with $\left[\gamma_{-}{ }^{32} \mathrm{P}\right] \mathrm{ATP}$, two ${ }^{32} \mathrm{P}$-labeled bands of $\sim 40$ and $60 \mathrm{kDa}$ are observed on the autoradiograph (Fig. 1C). These two bands are absent when the immunoprecipitate is prepared from cells not transfected with dSlo (Fig. 1C) or when GST alone is polymerized in the gel (data not shown). This result demonstrates the presence in the dSlo immunoprecipitate of at 
A
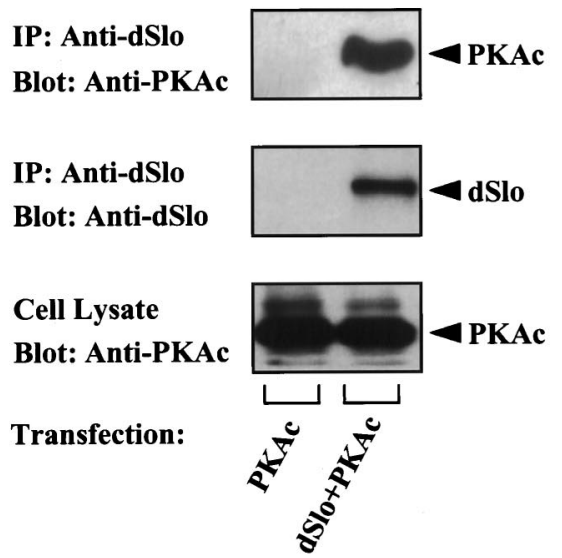

C

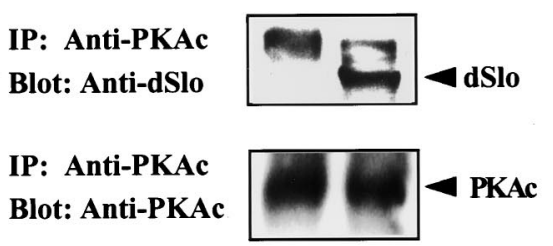

Cell Lysate
Blot: Anti-dSlo

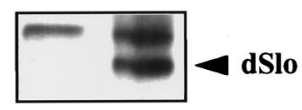

Fly genotype:
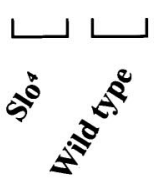

B
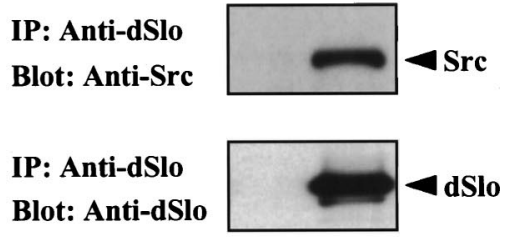

Cell Lysate

Blot: Anti-Src

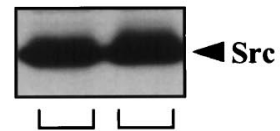

Transfection:

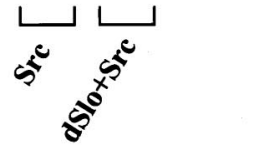

D

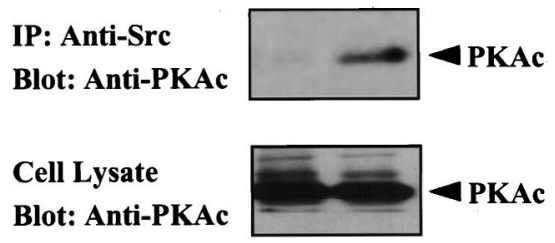

Cell Lysate

Blot: Anti-Src

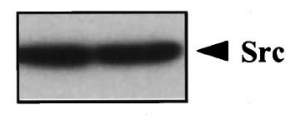

Transfection:

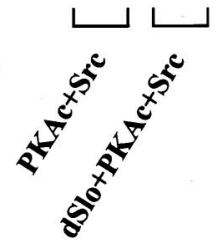

Figure 2. Binding of PKAc and Src to dSlo. $(A)$ Binding of PKAc to dSlo. Anti-dSlo immunoprecipitates prepared from cells transfected with PKAc alone or PKAc together with dSlo were analyzed on a Western blot probed with antiPKAc antibody (top panel). A parallel blot probed with anti-dSlo antibody shows the amount of dSlo protein in the immunoprecipitates (middle panel). The same amount of PKAc is expressed under the two transfection conditions (bottom panel). (B) Binding of Src to dSlo. Anti-dSlo immunoprecipitates prepared from cells transfected with Src alone or Src together with dSlo were analyzed on a Western blot probed with anti-Src antibody (top panel). A parallel blot probed with anti-dSlo antibody shows the amount of dSlo protein in the immunoprecipitates (middle panel). The same amount of Src is expressed under the two transfection conditions (bottom panel). (C) Binding of native PKAc and native dSlo in Drosophila. Anti-PKAc immunoprecipitates, prepared from either wildtype flies (right lane), or $\mathrm{slo}^{4}$ homozygous mutant flies that lack dSlo protein (left lane), were analyzed on a Western blot probed with anti-dSlo antibody (top panel). The reverse experiment (immunoprecipitate with anti-dSlo, probe with anti-PKAc) cannot be done because of the relatively small amount of PKAc in native tissue. PKAc immunoprecipitation (middle panel) and dSlo expression (bottom panel) are also assayed. (D) Simultaneous binding of PKAc and Src to dSlo. Anti-Src immunoprecipitates prepared from cells transfected with PKAc and Src or PKAc and Src together with dSlo were analyzed on a Western blot probed with anti-PKAc antibody (top panel). Anti-PKAc and anti-Src immunoblots of the cell lysates (middle and bottom panels) show that kinase expression is not influenced by coexpression of dSlo. least two protein kinases of these molecular weights, which are capable of phosphorylating GST-A in the gel.

\section{PKAc and Src coimmunoprecipitate with dSlo}

The molecular weights of PKAc and the Src tyrosine kinase are $\sim 40$ and $60 \mathrm{kDa}$, respectively. We therefore used antibodies against PKAc and Src to test for kinase immunoreactivities in the dSlo immunoprecipitate. PKAc is detected readily in a dSlo immunoprecipitate from tsA201 cells if the two proteins are coexpressed but not if PKAc is expressed alone (Fig. 2A, top panel). Precisely the same result is found for Src (Fig. 2B, top panel). In the reciprocal experiment, dSlo can be detected in an Src immunoprecipitate (data not shown). Thus both PKAc and Src can coimmunoprecipitate with dSlo in transfected cells. To determine whether the native dSlo channel can interact with native PKAc, the kinase was immunoprecipitated from Drosophila heads, and the immunoprecipitate was probed for dSlo on a Western blot. As shown in Figure $2 C$, a protein in the PKAc immunoprecipitate corresponding in molecular weight to dSlo is recognized by the dSlo antibody (top panel, right lane). When the immunoprecipitate is prepared from slo $^{4}$ mutant flies that lack dSlo (Atkinson et al., 1991), this band is not present (Fig. 2C, top panel, left lane), confirming that it is indeed the dSlo channel. These results provide strong evidence that a dSlo-PKAc complex exists in native Drosophila tissue.

\section{PKAc and Src bind simultaneously to dSlo}

To test whether the two kinases might interact simultaneously with dSlo, we coexpressed them, with or without the channel, and then immunoprecipitated Src with anti-Src antibody and tested for the coimmunoprecipitation of PKAc. No PKAc is detected in the anti-Src immunoprecipitate in the absence of dSlo (Fig. 2D, top panel, left lane), even when both PKAc and Src are expressed at high levels (Fig. 2D, middle and bottom panels); thus there is no direct interaction between the two kinases. However, when dSlo is also expressed, PKAc is present in the anti-Src immunoprecipitate (Fig. 2D, top panel, right lane). These results demonstrate clearly the existence of a complex in which PKAc and Src are bound simultaneously to dSlo.

\section{dSlo is phosphorylated by Src and PKAc}

The dSlo sequence contains several possible phosphorylation sites for Src. By using an antibody specific for phosphotyrosine, we found that dSlo is phosphorylated on tyrosine residues by endogenous kinase activity when the channel is expressed in tsA201 cells (Fig. 3A, top panel, left lane). Coexpression of the constitutively active Src kinase dramatically increases the tyrosine phosphorylation of dSlo (Fig. $3 A$, top panel, middle lane). Native dSlo immunoprecipitated from Drosophila heads is also phosphorylated on tyrosine residues by endogenous kinase activity (Fig. $3 \mathrm{~A}$, top panel, right lane). 


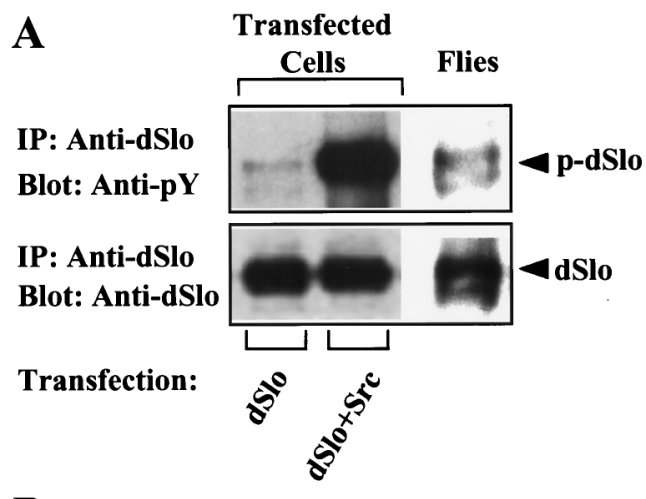

B

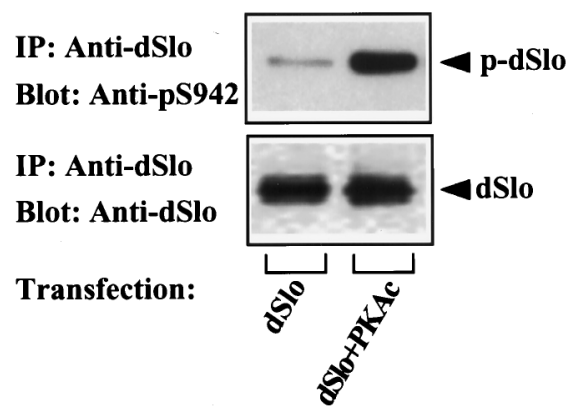

C IP from flies: Anti-dSlo

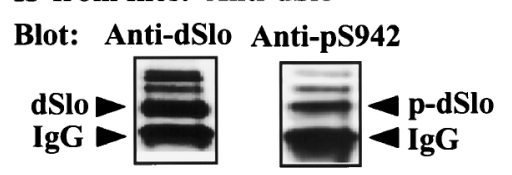

Figure 3. Phosphorylation of dSlo by Src and PKAc. $(A)$ Tyrosine phosphorylation of dSlo. Anti-dSlo immunoprecipitates prepared from Drosophila (right lane) or from tsA201 cells transfected with dSlo alone (left lane) or dSlo together with Src (middle lane) were analyzed on parallel Western blots probed with anti-phosphotyrosine (anti-pY) antibody (top panel) or anti-dSlo antibody (bottom panel). (B) Phosphorylation of S942 in dSlo-transfected cells. Anti-dSlo immunoprecipitates prepared from tsA201 cells transfected with dSlo alone (left lane) or dSlo together with PKAc (right lane) were analyzed on parallel Western blots probed with anti-pS942 (top panel) or anti-dSlo (bottom panel). (C) Phosphorylation of S942 in flies. Anti-dSlo immunoprecipitates prepared from Drosophila were analyzed on parallel Western blots probed with anti-dSlo (left panel) or anti-pS942 (right panel). In addition to the 130 $\mathrm{kDa}$ band that corresponds in molecular weight to dSlo (arrows), higher molecular weight bands that may represent aggregated or nondissociated channel subunits are recognized by both anti-dSlo and anti-pS942.

We also searched the dSlo sequence for possible PKA phosphorylation sites and found only one consensus site at serine 942 (S942). To determine whether this consensus site is subject to phosphorylation in vivo, we generated an antibody (anti-pS942) against a peptide, corresponding to a 10 -amino acid sequence flanking this PKA consensus site, with S942 thiophosphorylated enzymatically. This antibody is specific for dSlo phosphorylated on S942 (Wang, Zhou, Wen, and Levitan, manuscript in preparation). Using this antibody, we found that dSlo is phosphorylated on S942 by endogenous protein kinase activity in the tsA201 cells (Fig. 3B, top panel, left lane). Co-expression of PKAc with dSlo in tsA201 cells results in a large increase in the phosphorylation of S942 (Fig. 3B, top panel, right lane). Native dSlo immunoprecipitated from Drosophila heads (Fig. 3C, left lane) is also phosphorylated on $\mathrm{S} 942$ by endogenous protein kinase activity (Fig. 3C, right lane). Thus both protein kinases that coimmunoprecipitate with dSlo can phosphorylate the channel in native Drosophila tissue as well as in transfected cells.

\section{PKAc binds directly to a portion of the dSlo C-terminal tail}

Biochemical evidence for the direct association of protein tyrosine kinases with ion channels has been reported (Swope and Huganir, 1994; Fuhrer and Hall, 1996; Holmes et al., 1996; Yu et al., 1997). In contrast, PKAc generally is thought to be targeted to substrates indirectly, via its interaction with regulatory subunit and anchoring proteins (Mochly-Rosen, 1995; Faux and Scott, 1996). To test the possibility that PKAc binds to the dSlo channel directly, we biotinylated PKAc and used it to probe GST-dSlo fusion proteins immobilized on nitrocellulose membranes (overlay assay). Figure $4 A$ is an anti-GST immunoblot to identify the fusion proteins used in the overlay assay. GST-Cter is a $116-\mathrm{kDa}$ fusion protein containing the entire C-terminal domain of dSlo (from amino acid residues 337 to 1164). GST-A (46 kDa) and GST-C (48 kD) contain parts of the C-terminal domain of dSlo from residues 821 to 993 and 502 to 686, respectively. Note that there is much more GST protein than GST fusion proteins in the gel (Fig. $4 A$ ), yet biotinylated PKAc cannot bind to GST alone in the overlay assay (Fig. 4B, lane 1). However, PKAc can bind to GST-Cter (Fig. 4B, lane 4), indicating that PKAc and the dSlo tail can associate with each other directly. Furthermore, PKAc can bind to GST-A (Fig. 4B, lane 2) but not to GST-C (Fig. 4B, lane 3 ) in the overlay assay. These data demonstrate that PKAc binds directly to a discrete region in the $\mathrm{C}$-terminal domain of dSlo, suggesting the existence of a novel mechanism for the targeting of this kinase. Although S942 lies within this region of the channel, its phosphorylation does not appear to be required for PKAc binding, because the kinase can bind equally well in the overlay assay to S942A GST-A (Fig. 4C).

\section{Phosphorylatable tyrosines in dSlo are important for Src binding}

dSlo contains several tyrosine residues that can be phosphorylated by Src, including Y552 and Y976 in the C-terminal domain of the channel (Wang, Zhou, Wen, and Levitan, manuscript in preparation). To determine whether these might be required for Src binding, we expressed either wild-type or double-mutant Y552F/Y976F dSlo together with Src and immunoprecipitated the kinase. As shown in Figure $4 D$, there is far more wild-type (top panel, right lane) than mutant (top panel, left lane) dSlo in the Src immunoprecipitate, although both channels are expressed to the same extent (Fig. 4D, bottom panels). These results suggest that tyrosine phosphorylation of dSlo may enhance its binding to Src, possibly via a direct Src homology region 2 (SH2) domain interaction.

\section{Effects of bound kinases on dSlo}

dSlo current was measured in detached membrane patches to determine whether channel activity is influenced by coexpression of Src or PKAc. Somewhat surprisingly, we observed no obvious differences in the peak current amplitude $(621 \pm 510 \mathrm{pA} / \mathrm{patch}$ in cells transfected with dSlo alone; $622 \pm 308 \mathrm{pA} /$ patch in kinase cotransfected cells; mean $\pm \mathrm{SE})$ or voltage dependence $\left(\mathrm{V}_{1 / 2}\right.$ at 30 $\mu \mathrm{M}$ free calcium, $26 \pm 4 \mathrm{mV}$ in dSlo-transfected cells; $28 \pm 4 \mathrm{mV}$ in kinase cotransfected cells) of dSlo current after coexpression with the two protein kinases under these experimental conditions. However, the subcellular distribution of channel protein appears 
A

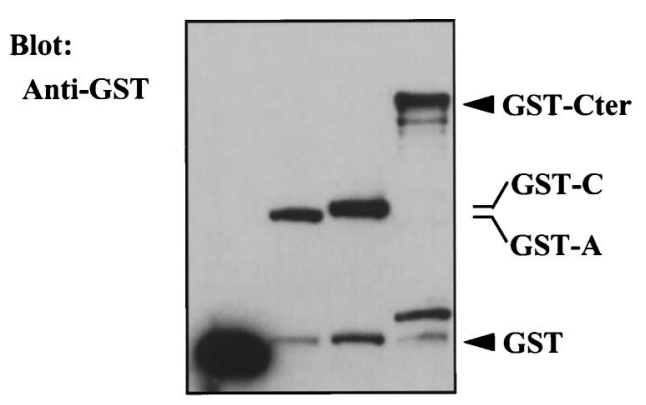

C

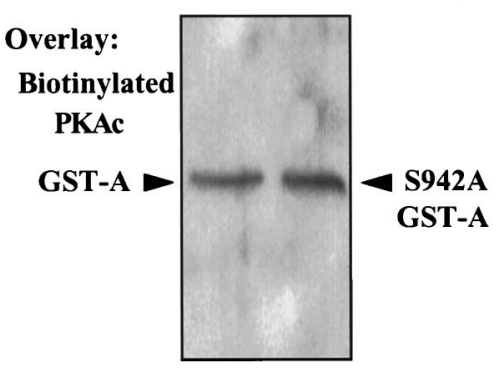

Fusion proteins:

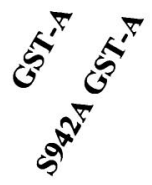

D

B

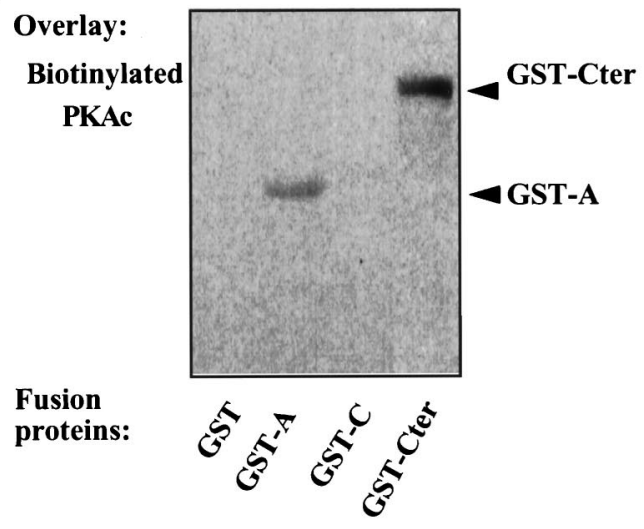

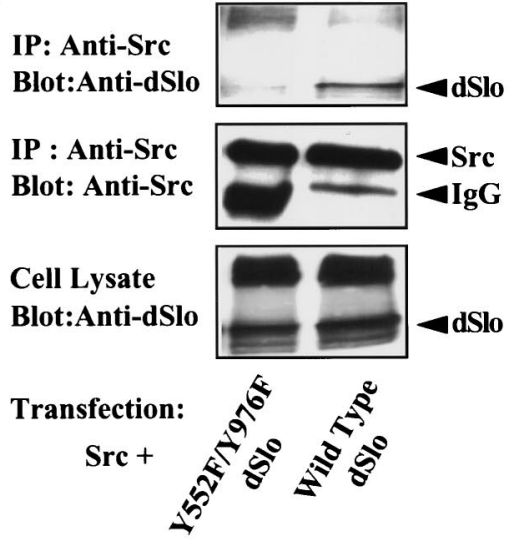

Figure 4. Direct binding of kinases to dSlo. $A$, Anti-GST blot of GST alone and GSTdSlo fusion proteins. $B, C$, Protein overlay assays. Biotinylated PKAc was used to probe GST fusion proteins immobilized on a nitrocellulose membrane. $B$, PKAc binds selectively to GST-A. $C$, PKAc binds equally well to wild-type and S942A GST-A. D, Binding of wild-type and mutant dSlo to Src. Anti-Src immunoprecipitates prepared from cells transfected with Src and wild-type dSlo (right lane) or Src and Y552F/Y976F mutant dSlo (left lane) were analyzed on a Western blot probed with anti-dSlo antibody (top panel). The middle and bottom panels show that the Src immunoprecipitation and dSlo expression are similar with the wild-type and mutant channels. to be different when the channel is coexpressed with either Src or PKAc (Wang, Zhou, Wen, and Levitan, manuscript in preparation). The significance of this apparent change in channel localization remains to be determined.

\section{DISCUSSION}

\section{A channel-kinase complex}

Much of the evidence for the intimate functional association of protein kinases with native ion channels has been inference based on electrophysiological analysis (Chung et al., 1991; White et al., 1993; Bielefeldt and Jackson, 1994; Lee et al., 1995; Reinhart and Levitan, 1995; Tian et al., 1998). In addition, our previous suggestion, based on electrophysiological studies in Xenopus oocytes, that a PKA-like kinase might associate with cloned dSlo channels (Esguerra et al., 1994), remains questionable (Bowlby and Levitan, 1996) because of the unusual variability of dSlo channel activity in oocytes (Bowlby and Levitan, 1996; Silberberg et al., 1996). However, more recent biochemical experiments have demonstrated unequivocally the direct binding of protein kinases (Swope and Huganir, 1994; Fuhrer and Hall, 1996; Holmes et al., 1996; Yu et al., 1997) and other signaling proteins (Schopperle et al., 1998; Xia et al., 1998) to ion channels, and the present results definitively extend direct biochemical evidence for channel-kinase association to the family of Slowpoke large-conductance calcium-dependent potassium channels. The domains that mediate the binding of Src to dSlo remain to be identified. Src can bind to the Kv1.5 voltage-dependent potassium channel via a direct interaction between the $\mathrm{SH} 3$ domain in Src and a proline-rich motif in the channel (Holmes et al., 1996), but the dSlo sequence
(Atkinson et al., 1991; Adelman et al., 1992) does not contain any obvious proline-rich motif that might bind to an SH3 domain. It is conceivable that tyrosine-phosphorylated dSlo binds to the Src $\mathrm{SH} 2$ domain, as is the case for the association of the nicotinic acetylcholine receptor from Torpedo with the Fyn and Fyk tyrosine kinases (Swope and Huganir, 1994). This is consistent with the present finding that two phosphorylatable tyrosine residues in dSlo are important for Src binding. Alternatively, the dSlo-Src association might be mediated by an adaptor protein (e.g., Colledge and Froehner, 1997) expressed in both tsA201 cells and Drosophila heads or by direct binding of specific sequences that involve neither the SH2 nor SH3 domain (e.g., Fuhrer and Hall, 1996; Yu et al., 1997).

\section{Direct binding of PKAc to dSlo}

In the case of PKAc, the overlay assay demonstrates clearly that the kinase binds directly to a specific region in the $\mathrm{C}$-terminal domain of dSlo. This is unexpected and interesting, because PKAc has not been shown previously to participate directly in a stable coimmunoprecipitating complex with one of its substrates. Rather, it has been known for some time that the PKA regulatory subunit and anchoring proteins that bind to it and other enzymes (Carr et al., 1992; Coghlan et al., 1995; Mochly-Rosen, 1995; Faux and Scott, 1996; Klauck et al., 1996) are important for targeting the kinase to specific substrates, including ion channels (Johnson et al., 1994; Rosenmund et al., 1994; Gao et al., 1997; Gray et al., 1997, 1998). Recently it was found that PKAc interacts with the $\mathrm{NF}-\kappa \mathrm{B}$ inhibitory protein $\mathrm{I} \kappa \mathrm{B}$, possibly by direct binding (Zhong et al., 1997). It is possible that direct sequence-specific binding is 
an alternative way to target this kinase to some subset of substrates that must be phosphorylated quickly in response to a cellular signal. It remains to be determined what signals influence the channel-kinase complex in neurons, and how PKAc activity is regulated in the absence of the regulatory subunit (Yang et al., 1995). In preliminary experiments, we have found that PKAc can still phosphorylate Kemptide, a specific PKA substrate (Kemp and Pearson, 1990), even when the kinase is bound to GST-A (H. Wen and I. B. Levitan, unpublished results).

\section{Functional consequences of kinase binding to dSlo}

We were surprised to find no obvious differences in the biophysical properties of dSlo current when the channel was coexpressed with the protein kinases. One possible explanation for this finding is that the channels whose activity we are able to measure in the detached membrane patches are not bound to the kinases. Although most studies of channel phosphorylation have focused on the acute actions of protein kinases and phosphatases on channel functional properties (Levitan, 1994), it is important to consider the possibility that channel expression, processing, membrane targeting, and turnover may be additional potential modulatory targets for kinases.

\section{A specific channel-kinase regulatory complex}

Not all protein kinases bind to dSlo. For example, we find that CaMKII does not coimmunoprecipitate with dSlo, although it can phosphorylate the channel on S942 both in vitro and in vivo (Wen and Levitan, unpublished results). It is intriguing that human Slowpoke, which shares substantial sequence identity with dSlo, does bind to CaMKII (Reinhart et al., 1997; confirmed by us), suggesting that even closely related ion channels may be associated with different complements of signaling proteins. Particularly significant is the unexpected finding that dSlo can bind to two protein kinases simultaneously. One is a serine/threonine kinase, and the other is a tyrosine kinase, and they are subject to regulation by different signal transduction pathways. It will be interesting to determine whether channel-kinase association itself is subject to physiological control in neurons and whether the binding and/or the activity of each kinase can be regulated independently. These are compelling questions, because PKAc normally is constitutively active in the absence of its regulatory subunit. This demonstration that a single potassium channel can associate simultaneously with multiple protein kinases emphasizes the richness and complexity of the signal transduction pathways that can influence the properties of neuronal ion channels.

\section{REFERENCES}

Adelman JP, Shen K-Z, Kavanaugh MP, Warren RA, Wu Y-N, Lagrutta A, Bond CT, North RA (1992) Calcium-activated potassium channels expressed from cloned complementary DNAs. Neuron 9:209-216.

Atkinson NS, Robertson GA, Ganetzky B (1991) A component of calcium-activated potassium channels encoded by the Drosophila slo locus. Science 253:551-555.

Bielefeldt K, Jackson MB (1994) Intramolecular and intermolecular enzymatic modulation of ion channels in excised membrane patches. Biophys J 66:1904-1914.

Bowlby MR, Levitan IB (1996) Kinetic variability and modulation of $d S l o$, a cloned calcium-dependent potassium channel. Neuropharmacology $35: 867-875$.

Carr DW, Stofko-Hahn RE, Fraser IDC, Cone RD, Scott JD (1992) Localization of the cAMP-dependent protein kinase to the postsynaptic densities by A-kinase anchoring proteins. J Biol Chem 267:16816-16823.
Chen C, Pan B (1994) Oncogenic ras stimulates a 96-kD histone H2b kinase activity in activated Xenopus egg extract. J Biol Chem 269:28034-28043.

Chung SK, Reinhart PH, Martin BL, Brautigan D, Levitan IB (1991) Protein kinase activity closely associated with a reconstituted calciumactivated potassium channel. Science 253:560-562.

Coghlan VM, Perrino BA, Howard M, Langeberg LK, Hicks JB, Gallatin WM, Scott JD (1995) Association of protein kinase A and protein phosphatase 2B with a common anchoring protein. Science 267:108-111.

Colledge M, Froehner SC (1997) Tyrosine phosphorylation of nicotinic acetylcholine receptor mediates Grb2 binding. J Neurosci 17: $5038-5045$.

Esguerra M, Wang J, Foster CD, Adelman JP, North RA, Levitan IB (1994) Phosphorylation at a specific serine residue by a functionally associated protein kinase modulates a cloned calcium-dependent potassium channel. Nature 369:563-565.

Faux MC, Scott JD (1996) Molecular glue: kinase anchoring and scaffold proteins. Cell 85:9-12.

Fuhrer C, Hall ZW (1996) Functional interaction of Src family kinases with the acetylcholine receptor in $\mathrm{C} 2$ myotubes. J Biol Chem 271:32474-32481.

Gao T, Yatani A, Dell'Acqua ML, Sako H, Green SA, Dascal N, Scott JD, Hosey MM (1997) cAMP-dependent regulation of cardiac L-type $\mathrm{Ca}^{2+}$ channels requires membrane targeting of PKA and phosphorylation of channel subunits. Neuron 19:185-196.

Gray PC, Tibbs VC, Catterall WA, Murphy BJ (1997) Identification of a 15-kDa cAMP-dependent protein kinase-anchoring protein associated with skeletal muscle L-type calcium channels. J Biol Chem 272:6297-6302.

Gray PC, Johnson BD, Westenbroek RE, Hays LG, Yates JR, Scheuer T, Catterall WA, Murphy BJ (1998) Primary structure and function of an A kinase anchoring protein associated with calcium channels. Neuron 20:1017-1026.

Harlow E, Lane D (1988) Antibodies: a laboratory manual. New York: Cold Spring Harbor Laboratory.

Holmes TC, Fadool DA, Ren R, Levitan IB (1996) Association of Src tyrosine kinase with a human potassium channel mediated by SH3 domain. Science 274:2089-2091.

Johnson BD, Scheuer T, Catterall WA (1994) Voltage-dependent potentiation of L-type $\mathrm{Ca}^{2+}$ channels in skeletal muscle cells requires anchored cAMP-dependent protein kinase. Proc Natl Acad Sci USA 91:11492-11496.

Kemp BE, Pearson RB (1990) Protein kinase recognition sequence motifs. Trends Biochem Sci 15:342-346.

Klauck TM, Faux MC, Labudda K, Langeberg LK, Jaken S, Scott JD (1996) Coordination of three signaling enzymes by AKAP79, a mammalian scaffold protein. Science 271:1589-1592.

Latorre R, Oberhauser A, Labarca P, Alvarez O (1989) Varieties of calcium-activated potassium channels. Annu Rev Physiol 51:385-399.

Lee K, Rowe ICM, Ashford MLJ (1995) Characterization of an ATPmodulated large conductance $\mathrm{Ca}^{2+}$-activated $\mathrm{K}^{+}$channel present in rat cortical neurones. J Physiol (Lond) 488:319-337.

Levitan IB (1994) Modulation of ion channels by protein phosphorylation and dephosphorylation. Annu Rev Physiol 56:193-212.

Margolskee RF, McHendry-Rinde B, Horn R (1993) Panning transfected cells for electrophysiological studies. Biotechniques 15:906-911.

Mochly-Rosen D (1995) Localization of protein kinases by anchoring proteins: a theme in signal transduction. Science 268:247-251.

Park CS, Hausdorff SF, Miller C (1991) Design, synthesis, and functional expression of a gene for charybdotoxin, a peptide blocker of $\mathrm{K}^{+}$ channels. Proc Natl Acad Sci USA 88:2046-2050.

Patton BL, Miller SG, Kennedy MB (1991) Identification of functionally significant phosphorylation sites on neuronal proteins and preparation of antibodies that recognize them. Methods Neurosci 6:158-176.

Reinhart PH, Levitan IB (1995) Kinase and phosphatase activities intimately associated with a reconstituted calcium-dependent potassium channel. J Neurosci 15:4572-4579.

Reinhart PH, Buckholz R, Estey L (1997) Calcium calmodulin kinase II forms a protein complex with hSla $\mathrm{Ca}^{++}$activated $\mathrm{K}^{+}$channels. Soc Neurosci Abstr 23:1737

Rosenmund C, Carr DW, Bergeson SE, Nilaver G, Scott JD, Westbrook GL (1994) Anchoring of protein kinase A is required for modulation of AMPA/kainate receptors on hippocampal neurons. Nature 368:853-856. 
Sambrook J, Fritsch EF, Maniatas T (1989) Molecular cloning: a laboratory manual. New York: Cold Spring Harbor Laboratory.

Schopperle WM, Holmqvist MH, Zhou Y, Wang J, Wang Z, Griffith LC, Keselman I, Kusinitz F, Dagan D, Levitan IB (1998) Slob, a novel protein that interacts with the slowpoke calcium-dependent potassium channel. Neuron 20:565-573.

Silberberg SD, Lagrutta A, Adelman JP, Magleby KL (1996) Wanderlust kinetics and variable $\mathrm{Ca}^{2+}$-sensitivity of Drosophila, a large conductance $\mathrm{Ca}^{2+}$-activated $\mathrm{K}^{+}$channel, expressed in oocytes. Biophys $\mathrm{J}$ 70:2640-2651.

Swope SL, Huganir RL (1994) Binding of the nicotinic acetylcholine receptor to SH2 domains of Fyn and Fyk protein tyrosine kinases. J Biol Chem 269:29817-29824.

Tian L, Knaus H-G, Shipston MJ (1998) Glucocorticoid regulation of calcium-activated potassium channels mediated by serine/threonine protein phosphatase. J Biol Chem 273:13531-13536.

White RE, Lee AB, Shcherbatko AD, Lincoln TM, Schonbrunn A,
Armstrong DL (1993) Potassium channel stimulation by natriuretic peptides through cGMP-dependent dephosphorylation. Nature 361:263-266.

Xia X-M, Hirschberg B, Smolik S, Forte M, Adelman JP (1998) dSlo interacting protein 1 , a novel protein that interacts with largeconductance calcium-activated potassium channels. J Neurosci 18:2360-2369.

Yang S, Fletcher WH, Johnson DA (1995) Regulation of cAMPdependent protein kinase: enzyme activation without dissociation. Biochemistry 34:6267-6271.

Yu X-M, Askalan R, Keil GJ, Salter MW (1997) NMDA channel regulation by channel-associated protein tyrosine kinase Src. Science 275:674-678.

Zhong H, SuYang H, Erdjument-Bromage H, Tempst P, Ghosh S (1997) The transcriptional activity of NF-kB is regulated by the IkBassociated PKAc subunit through a cyclic AMP-independent mechanism. Cell 89:413-424. 